\title{
High frequency of low-virulent microorganisms detected by sonication of pedicle screws: a potential cause for implant failure
}

\author{
Vincent Prinz, MD, ${ }^{1}$ Simon Bayerl, MD, ${ }^{1}$ Nora Renz, MD, ${ }^{2}$ Andrej Trampuz, MD, ${ }^{2}$ \\ Marcus Czabanka, MD, ${ }^{1}$ Johannes Woitzik, MD, ${ }^{1}$ Peter Vajkoczy, MD, ${ }^{1}$ and Tobias Finger, MD${ }^{1}$ \\ ${ }^{1}$ Department of Neurosurgery and ${ }^{2}$ Center for Musculoskeletal Surgery, Charité-Universitätsmedizin Berlin, Corporate Member of \\ Freie Universität Berlin, Humboldt-Universität zu Berlin, and Berlin Institute of Health, Berlin, Germany
}

\begin{abstract}
OBJECTIVE Loosening of pedicle screws is a frequent complication after spinal surgery. Implant colonization with lowvirulent microorganisms forming biofilms may cause implant loosening. However, the clinical evidence of this mechanism is lacking. Here, the authors evaluated the potential role of microbial colonization using sonication in patients with clinical pedicle screw loosening but without signs of infection.
\end{abstract}

METHODS All consecutive patients undergoing hardware removal between January 2015 and December 2017, including patients with screw loosening but without clinical signs of infection, were evaluated. The removed hardware was investigated using sonication.

RESULTS A total of 82 patients with a mean ( \pm SD) patient age of $65 \pm 13$ years were eligible for evaluation. Of the 54 patients with screw loosening, 22 patients $(40.7 \%)$ had a positive sonication result. None of the 28 patients without screw loosening who served as a control cohort showed a positive sonication result $(p<0.01)$. In total, 24 microorganisms were detected in those 22 patients. The most common isolated microorganisms were coagulase-negative staphylococci (62.5\%) and Cutibacterium acnes (formerly known as Propionibacterium acnes) (25\%). When comparing only the patients with screw loosening, the duration of the previous spine surgery was significantly longer in patients with a positive microbiological result ( $288 \pm 147$ minutes) than in those with a negative result ( $201 \pm 103$ minutes) $(p=0.02)$.

CONCLUSIONS The low-virulent microorganisms frequently detected on pedicle screws by using sonication may be an important cause of implant loosening and failure. Longer surgical duration increases the likelihood of implant colonization with subsequent screw loosening. Sonication is a highly sensitive approach to detect biofilm-producing bacteria, and it needs to be integrated into the clinical routine for optimized treatment strategies.

https://thejns.org/doi/abs/10.3171/2019.1.SPINE181025

KEYWORDS pedicle screw loosening; infection; biofilm; complications; failed back surgery; sonication; antimicrobial treatment

$\mathrm{P}$ OSTERIOR instrumentation using pedicle screws is a widely used and well-established technique to stabilize the thoracolumbar as well as cervical spine for a variety of indications. Using pedicle screws, immediate and strong fixation is achieved after spinal decompression as well as in the surgical treatment of spinal deformities or treatment of vertebral fractures. As shown by meta-analysis, the additional use of intraoperative 3D imaging and navigation has helped to further improve pedicle screw accuracy in all regions of the spine, thereby potentially reducing reoperation rates and further exposure to radiation and anesthesia. ${ }^{6,8}$ Loosening of pedicle screws associated with cage dislocation or vertebral fracture at the level of the screw is one of the most common complications after spinal posterior instrumentation., ${ }^{7,14}$ The percentage of pedicle screw loosening and instrumentation-associated vertebral fracture is estimated to range from less than $1 \%$ to $15 \%$ in nonosteoporotic patients; in osteoporotic patients, the rate of pedicle screw loosening is even higher, reaching up to $60 \%$. $1,5,7,12$

Spinal surgery in osteoporotic patients is becoming more frequent due to a growing aging population., ${ }^{1,11}$ Pedicle screw loosening often leads to loss of spinal stability, resulting in pseudarthrosis and consecutive pain as well 
as recurrent spinal stenosis and back pain, which can occur even in fused segments. Extensive revision surgery to replace the loosened screws, along with extension of the construct and augmentation techniques, is the most often performed procedure for symptomatic loosened pedicle screws.

Pedicle screw loosening can be related to a number of different factors, such as exposing bone to mechanical stress due to excessive loading as well as sagittal imbalance, especially in obese patients. ${ }^{7}$ In addition to the aforementioned mechanisms, it has been suggested that implant-associated infection is related to screw loosening. However, clinical evidence about this possible mechanism is scarce, despite its possibly enormous clinical importance. Implantable devices are highly susceptible to bacterial colonization; even low numbers of bacteria can cause infection with a delayed clinical onset. Microorganisms typically adhere to the surface of the device and form biofilms, making them difficult to detect by conventional methods. ${ }^{3,13}$ Sonication was specifically designed for the detection of microbial biofilms, which can be found in the sonication fluid after being released from the implant surface. In fact, in orthopedic prostheses, the use of sonication has demonstrated higher sensitivity and specificity in comparison with conventional tissue cultures. ${ }^{2,13}$

In this prospective cohort study, we used sonication to evaluate the possible microbial colonization of loosened pedicle screws as a potential cause of implant failure.

\section{Methods Study Design}

This prospective observational study was conducted in a tertiary healthcare center that provides advanced specialty care to a population of about 4 million inhabitants. The study was approved by the local ethics committee and was conducted in accordance with the Declaration of Helsinki. Patient consent was not required for this prospective observational study, as sonication is performed as part of the routine microbiological investigation.

\section{Study Population}

A total of 269 consecutive adult patients in whom spinal implants were removed during a revision surgery between January 1, 2015, and December 31, 2017, were screened. Patients with screw loosening and/or cage dislocation due to vertebral metastases, spondylodiscitis, paravertebral abscesses, or the intraoperative finding of infection were excluded (Table 1 and Fig. 1).

\section{Data Collection}

Patient data were collected using a standardized case report form. All patients were evaluated by an interdisciplinary team that included spine surgeons and infectious disease specialists. The following variables were extracted: age, sex, height, weight, BMI, screw loosening, number of stabilized spinal units, duration of the previous surgery, blood transfusion during the previous surgery, comorbidities, and microbiological results of the sonication fluid. Growth in the sonication fluid culture was defined as a positive microbiological result.

\section{Surgical Procedure}

All surgical procedures were performed under sterile conditions in the operating room with the patient under general anesthesia. The screw and rod systems were removed and immediately placed in an airtight container to minimize the risk of contamination; then they were sent for analysis. Perioperative antibiotics (cefazolin) were administered intravenously in all patients 30-60 minutes before skin incision.

\section{Definition of Screw Loosening}

All patients were preoperatively assessed using CT scanning to see if the screws were solidly anchored to the pedicles or if they were loosened. We did not rely on radiographs, as a study comparing CT scans and standard radiographs showed a significantly lower sensitivity of the latter assessment method when compared with the former. ${ }^{9}$ All CT scans were evaluated by at least 1 radiologist and 2 spine surgeons.

\section{Sonication of Removed Implants}

The removed hardware was transported to the microbiological laboratory in a sterile airtight container (Lock \& Lock). Sonication was performed within 6 hours of removal. After the addition of $5 \mathrm{ml}$ of normal saline covering the implant, the container was vortexed for 30 seconds, sonicated for 1 minute at $40 \mathrm{kHz}$ (BactoSonic, Bandelin), and vortexed for another 30 seconds. The resulting sonication fluid was processed as described above for conventional cultures. Microorganisms on plates were enumerated (i.e., number of colony-forming units/ml sonication fluid) and identified using routine microbiological techniques. Contaminants were excluded from the analysis.

\section{Statistical Analysis}

ANOVA was used to test differences in continuous variables. Mean values are reported with standard deviations. All statistical tests were two-tailed, and statistical significance was set at $\mathrm{p}<0.05$. IBM SPSS software (version 21, IBM Corp.) was used for all analyses.

\section{Results \\ Demographic Data}

We screened 269 patients in whom spinal implants were removed during surgery between January 1, 2015, and December 31, 2017. Patients with preoperative MRI or CT evidence of spondylodiscitis, evidence of a systemic infection (clinical and/or laboratory findings), or intraoperative evidence of infection were excluded. Sonication analysis of the removed hardware was performed in the remaining 82 patients, and these patients were included in this study. None of the patients included in the analysis were on long-term antibiotic therapy prior to implant removal. Of these patients, no signs of screw loosening could be detected in 28 patients. The reasons for material removal were adjacent-segment disease $(n=13)$, adjacentsegment fracture $(n=3)$, cage dislocation $(n=2)$, artificial disc dislocation $(n=1)$, rod breakage $(n=5)$, and screw breakage $(n=4)$. We used these 28 patients as a control 
TABLE 1. Demographic data, information concerning the surgical procedure, duration of hospitalization, and laboratory methods and results

\begin{tabular}{|c|c|c|c|c|}
\hline & $\begin{array}{l}\text { All Patients w/ Sonication } \\
\text { Analysis }(n=82)\end{array}$ & $\begin{array}{c}\text { Sonication } \\
\text { Positive }(n=22)\end{array}$ & $\begin{array}{c}\text { Sonication } \\
\text { Negative }(n=60)\end{array}$ & $\begin{array}{c}\mathrm{p} \\
\text { Value }\end{array}$ \\
\hline Mean age, yrs & $65 \pm 13$ & $64 \pm 13$ & $65 \pm 13$ & 0.83 \\
\hline Mean height, $\mathrm{m}$ & $1.68 \pm 0.09$ & $1.71 \pm 0.08$ & $1.67 \pm 0.09$ & 0.13 \\
\hline Mean weight, kg & $77.8 \pm 15.8$ & $80.9 \pm 15.8$ & $76.7 \pm 15.8$ & 0.30 \\
\hline Mean BMI, kg/m² & $27.4 \pm 4.6$ & $27.5 \pm 4.9$ & $27.4 \pm 4.5$ & 0.90 \\
\hline Sex & & & & 0.06 \\
\hline Male & 31 & 12 & 19 & \\
\hline Female & 51 & 10 & 41 & \\
\hline Screw loosening & $65.9 \%$ & $100 \%$ & $53.3 \%$ & $<0.01$ \\
\hline \multicolumn{5}{|l|}{ Surgical procedure } \\
\hline Mean no. of stabilized motion segments & $3.5 \pm 3.3$ & $4.0 \pm 3.8$ & $3.3 \pm 3.1$ & 0.37 \\
\hline Mean no. of previous spine surgeries & $1.7 \pm 1.1$ & $1.8 \pm 1.3$ & $1.7 \pm 1.0$ & 0.72 \\
\hline $\begin{array}{l}\text { Mean duration of previous spine surgery, } \\
\text { mins }\end{array}$ & $220 \pm 118$ & $288 \pm 147$ & $201 \pm 103$ & 0.02 \\
\hline Transfusion during previous spine surgery & & & & 0.45 \\
\hline Yes & 10 & 3 & 7 & \\
\hline No & 47 & 9 & 38 & \\
\hline \multicolumn{5}{|l|}{ Comorbidities } \\
\hline Diabetes mellitus type 2 & $25.6 \%$ & $13.6 \%$ & $30.0 \%$ & 0.14 \\
\hline Smoking & $32.9 \%$ & $36.4 \%$ & $31.7 \%$ & 0.69 \\
\hline Alcohol abuse & $4.9 \%$ & $9.1 \%$ & $3.3 \%$ & 0.29 \\
\hline Preexisting oncological disease & $17.1 \%$ & $13.6 \%$ & $18.3 \%$ & 0.62 \\
\hline Radiation therapy & $2.4 \%$ & $4.5 \%$ & $1.7 \%$ & 0.46 \\
\hline Cortisol medication & $11.0 \%$ & $13.6 \%$ & $10.0 \%$ & 0.65 \\
\hline \multicolumn{5}{|l|}{ Laboratory values at admission } \\
\hline Mean white blood cell count/nl & $8.2 \pm 2.5$ & $8.9 \pm 2.6$ & $8.0 \pm 2.5$ & 0.13 \\
\hline Mean C-reactive protein, mg/L & $10.4 \pm 14.8$ & $9.6 \pm 12.0$ & $10.7 \pm 15.8$ & 0.76 \\
\hline
\end{tabular}

Mean values are presented with standard deviations. Boldface type indicates statistical significance.

group. All sonication analyses in these patients showed no evidence of pathogens. In the other 54 patients, screw loosening could be detected. The mean time between surgery and screw loosening in all patients combined was $606 \pm 903$ days (minimum 27 days, maximum 4550 days).

The mean patient age was $65 \pm 13$ years; their mean height and weight were $1.68 \pm 0.09 \mathrm{~cm}$ and $77.8 \pm 15.8$ $\mathrm{kg}$ with a BMI of $27.4 \pm 4.6$. Of the 82 patients, 51 were female $(62 \%)$.

We divided all 82 patients into 2 groups: 1) sonication positive and 2) sonication negative. The demographic data were similar with regard to age, sex, comorbidities, and laboratory values at admission in both groups. The predominant indication for the initial spinal stabilization surgery was degenerative spine disease (71 patients), vertebral osteomyelitis (6 patients), and spinal trauma (5 patients). Eighteen patients had their previous surgery in another department, of whom 10 had a positive microbiological result $(56 \%)$ (Table 1$)$.

\section{Surgical Procedure}

All patients received a single dose of perioperative antibiotics 30 minutes before skin incision. On average, each patient had $1.7 \pm 1.1$ previous surgeries (maximum 6 surgeries, minimum 1 surgery, mean 1 surgery). The average number of stabilized motion segments in all patients was $3.5 \pm 3.3$. No differences between the groups were detected $(\mathrm{p}=0.37)$. In 5 patients, a blood transfusion had to be given in the preceding surgery. There was no significant difference between groups $(\mathrm{p}=0.45)$.

When comparing the duration of the previous spine surgery, we found a significant difference between patients with a positive microbiological result (mean 288 \pm 147 minutes) and a negative result (mean $201 \pm 103$ minutes) $(\mathrm{p}=0.02)$. In patients with a longer duration of surgery (on average 92 minutes longer), screw loosening was more likely to be associated with a positive microbiological result (Table 1). In the subgroup analysis dividing the patients with screw loosening into 2 groups based on the sonication results, the duration of the previous surgery remained significantly longer in the sonication-positive group (p < 0.05) (Supplemental Table 1).

\section{Microbiological Findings}

The total number of detected pathogens was 24 . The most common pathogens were coagulase-negative staphy- 


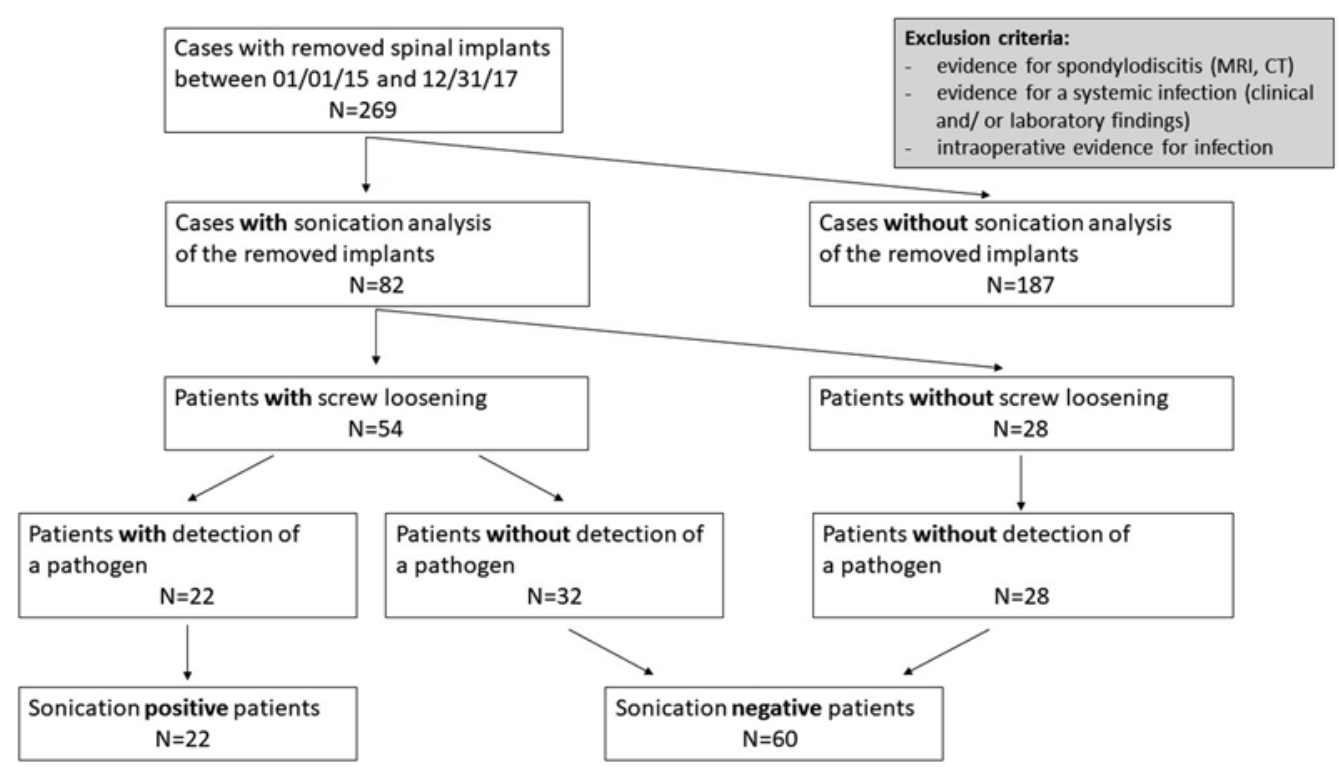

FIG. 1. Flowchart showing patient selection.

lococci (15 pathogens, 62.5\%) and Cutibacterium acnes (formerly known as Propionibacterium acnes) (6 pathogens, 25\%). In 20 patients a single pathogen was found, and in 2 patients 2 pathogens could be detected (Table 2).

\section{Interrelation Between Screw Loosening and Sonication Result}

All 22 patients with a positive sonication result experienced screw loosening (100\%), whereas in the 60 patients with a negative sonication result, only 32 patients showed signs of screw loosening $(53 \%)(\mathrm{p}<0.01)$.

If screw loosening was evident on the preoperative $\mathrm{CT}$ scans (54 patients), the chances of a positive sonication result with the detection of a pathogen was $40.7 \%$. In the 28 patients without screw loosening, none had a positive microbiological result (Table 3).

\section{Discussion}

This study has the following main findings. First, pedi-

TABLE 2. Frequency of isolated pathogens

\begin{tabular}{|c|c|c|c|}
\hline \multirow[b]{2}{*}{ Pathogens } & \multirow[b]{2}{*}{$\begin{array}{l}\text { Total } \\
\text { No. }\end{array}$} & \multicolumn{2}{|c|}{ Sonication } \\
\hline & & Positive & $\begin{array}{c}\text { Detection } \\
\text { Rate }\end{array}$ \\
\hline \multicolumn{4}{|l|}{ Low-virulent pathogens } \\
\hline Coagulase-negative staphylococci* & 15 & 15 & $100 \%$ \\
\hline Cutibacterium acnes & 6 & 6 & $100 \%$ \\
\hline \multicolumn{4}{|l|}{ High-virulent pathogens } \\
\hline Enterococcus faecalis & 1 & 1 & $100 \%$ \\
\hline Streptococcus parasanguinis & 2 & 2 & $100 \%$ \\
\hline
\end{tabular}

* Staphylococcus epidermidis $(\mathrm{n}=9)$, Staphylococcus hominis $(\mathrm{n}=2)$,

Staphylococcus lugdunensis $(n=1)$, Staphylococcus haemolyticus $(n=1)$,

Staphylococcus cohnii $(n=1)$, Staphylococcus saprophyticus $(n=1)$. cle screw loosening is significantly associated with a positive microbiological yield for low-virulent pathogens. Second, the most common pathogens were coagulase-negative staphylococci. Third, standard preoperative laboratory results do not reflect possible implant infection. Fourth, prolonged surgery time is significantly associated with a higher rate of implant infection.

Pedicle screw loosening is a frequent and important complication after spinal instrumentation. Device-associated infection has been suggested to be related to pedicle screw loosening.? Although implants are highly susceptible to bacterial colonization and even low numbers of bacteria can cause infection and implant failure, to date, clinical evidence about this possible mechanism is lacking.

In our study applying sonication to loosened pedicle screws in clinically aseptic patients, we detected a significant association with a positive microbiological yield for low-virulent pathogens. The finding that hardware infections may cause seemingly aseptic implant loosening is already known to be a problem in patients after knee and hip arthroplasty. ${ }^{4}$ However, despite its immense potential clinical impact, thus far, data concerning the association of pedicle screw loosening in spine surgery and infection are still surprisingly scarce. Our study clearly reveals infection of the implanted device with low-virulent patho-

TABLE 3. Interrelation between screw loosening and sonication results

\begin{tabular}{llccr}
\hline & & \multicolumn{2}{c}{ Sonication } & $p$ \\
\cline { 2 - 4 } & & Positive & Negative & Value \\
\hline \multirow{2}{*}{ Screw loosening } & Positive & $26.8 \%(22 \mathrm{pts})$ & $39.1 \%(32 \mathrm{pts})$ & 0.14 \\
\cline { 2 - 4 } & Negative & $0 \%(0 \mathrm{pts})$ & $34.1 \%(28 \mathrm{pts})$ & $<0.01$ \\
\hline & p value & $<0.01$ & 0.42 & \\
\hline
\end{tabular}

Pts = patients. 
gens as a substantial cause of symptomatic implant failure. Our data show that $40.7 \%$ of the tested explanted screws had a positive microbiological result; of these, $100 \%$ of the patients showed positive evidence for infection in the sonication analysis. In a recent, but rather heterogeneous study, similar results were reported.9 The spectrum of microbiological pathogens was also comparable, with our most common species being coagulase-negative staphylococci $(53.1 \%$ in the publication) and $C$. acnes $(40.6 \%$ in the publication). In comparison with the already-published data, we showed that a prolonged surgery time seems to influence the likelihood of a positive result in the microbiological analysis: the longer the contact of the implants with the surroundings, the higher the risk of a low-level contamination resulting in a subclinical infection causing material loosening. In our study, we focused the analysis on patients with solid evidence of screw loosening to rule out additional confounders. The diagnosis of screw loosening was made in our 54 patients based on preoperative CT scanning results. We did not rely on radiographs because of the lower sensitivity of the method used to obtain them. However, in the recently published data by Leitner et al., only 23 patients were found to have screw loosening on radiographs; of these patients, CT scanning was performed in only $13 .{ }^{9}$ We believe that by only including patients with a CT scan and screw loosening, we increased the quality of our data due to a greater homogeneity of the analyzed patient cohort.

The assessment of the consequence of a positive microbiological result in patients who are initially thought to have aseptic failure is still problematic. ${ }^{4}$ In publications covering the treatment of prosthetic joint infections, great differences can be found when it comes to treatment recommendations. The spectrum ranges from 6 weeks of intravenous antibiotics over oral antimicrobial agents alone to no medical treatment at all. In a study by Marculescu et al., 3 patients (of 16) with positive evidence for infection (2 Cutibacterium sp. and 1 Enterococcus sp.) and loosening of a joint prosthesis received no postoperative antimicrobial treatment after revision surgery. These patients had a favorable outcome without recurrent infection or repeated loosening of the implants. ${ }^{10}$

\section{Conclusions}

Infections of spinal stabilization devices with lowvirulent pathogens with a smoldering dynamic may be a relevant cause of implant failure. Prolonged duration of prior surgery seems to increase the likelihood of implant colonization with consequential infection and screw loosening. The method of sonication is an adequate approach to detect biofilm-producing bacteria and can be implemented very easily into the clinical routine. Our data reveal a very important and, until now, not yet fully understood potential reason for implant failures after spinal instrumentation. We strongly believe that cases with positive culture and new screws in place may benefit from systemic biofilm-active antibiotic therapy to prevent new screw loosening. However, to date, the literature does not support or refute that hypothesis. Alternatively, local antibiotics may be applied, such as the antimicrobial coating of screws or application of local antimicrobial hydrogel or bone substitutes.

Our results call for an interventional study to prevent recurrence of infection. Antimicrobial strategies in the revision of loosened screws may have great impact in patients after spinal stabilization by reducing the need for subsequent surgeries, antibiotic exposure, and repeated hospital stays. Of course, potential adverse effects of systemic or local antibiotics need to be taken in account as well.

\section{Acknowledgments}

Dr. Prinz is a participant in the Charité Clinical Scientist Program funded by the Charité-Universitätsmedizin Berlin and the Berlin Institute of Health.

\section{References}

1. Burge R, Dawson-Hughes B, Solomon DH, Wong JB, King A, Tosteson A: Incidence and economic burden of osteoporosis-related fractures in the United States, 2005-2025. J Bone Miner Res 22:465-475, 2007

2. Conen A, Fux CA, Vajkoczy P, Trampuz A: Management of infections associated with neurosurgical implanted devices. Expert Rev Anti Infect Ther 15:241-255, 2017

3. Costerton JW, Stewart PS, Greenberg EP: Bacterial biofilms: a common cause of persistent infections. Science 284:13181322,1999

4. Del Pozo JL, Patel R: Clinical practice. Infection associated with prosthetic joints. N Engl J Med 361:787-794, 2009

5. El Saman A, Meier S, Sander A, Kelm A, Marzi I, Laurer $\mathrm{H}$ : Reduced loosening rate and loss of correction following posterior stabilization with or without PMMA augmentation of pedicle screws in vertebral fractures in the elderly. Eur J Trauma Emerg Surg 39:455-460, 2013

6. Fichtner J, Hofmann N, Rienmüller A, Buchmann N, Gempt J, Kirschke JS, et al: Revision rate of misplaced pedicle screws of the thoracolumbar spine-comparison of three-dimensional fluoroscopy navigation with freehand placement: a systematic analysis and review of the literature. World Neurosurg 109:e24-e32, 2018

7. Galbusera F, Volkheimer D, Reitmaier S, Berger-Roscher N, Kienle A, Wilke HJ: Pedicle screw loosening: a clinically relevant complication? Eur Spine J 24:1005-1016, 2015

8. Hecht N, Yassin H, Czabanka M, Föhre B, Arden K, Liebig $\mathrm{T}$, et al: Intraoperative computed tomography versus 3D $\mathrm{C}$-arm imaging for navigated spinal instrumentation. Spine (Phila Pa 1976) 43:370-377, 2018

9. Leitner L, Malaj I, Sadoghi P, Amerstorfer F, Glehr M, Vander K, et al: Pedicle screw loosening is correlated to chronic subclinical deep implant infection: a retrospective database analysis. Eur Spine J 27:2529-2635, 2018

10. Marculescu CE, Berbari EF, Hanssen AD, Steckelberg JM, Osmon DR: Prosthetic joint infection diagnosed postoperatively by intraoperative culture. Clin Orthop Relat Res 439:38-42, 2005

11. Melton LJ III: Epidemiology of spinal osteoporosis. Spine (Phila Pa 1976) 22 (24 Suppl):2S-11S, 1997

12. Okuyama K, Abe E, Suzuki T, Tamura Y, Chiba M, Sato K: Influence of bone mineral density on pedicle screw fixation: a study of pedicle screw fixation augmenting posterior lumbar interbody fusion in elderly patients. Spine J 1:402-407, 2001

13. Trampuz A, Piper KE, Jacobson MJ, Hanssen AD, Unni KK, Osmon DR, et al: Sonication of removed hip and knee prostheses for diagnosis of infection. N Engl J Med 357:654663,2007 
14. Young PM, Berquist TH, Bancroft LW, Peterson JJ: Complications of spinal instrumentation. Radiographics 27:775789,2007

\section{Disclosures}

This work was supported by an educational grant from the PROIMPLANT Foundation (https://www.pro-implant-foundation.org).

\section{Author Contributions}

Conception and design: Prinz, Finger. Acquisition of data: Prinz, Renz, Czabanka, Woitzik, Finger. Analysis and interpretation of data: Vajkoczy, Prinz, Bayerl, Trampuz, Finger. Drafting the article: Vajkoczy, Prinz, Trampuz, Finger. Critically revising the article: all authors. Reviewed submitted version of manuscript: Vajkoczy, Prinz, Finger. Approved the final version of the manuscript on behalf of all authors: Vajkoczy.

\section{Supplemental Information}

Online-Only Content

Supplemental material is available with the online version of the article.

Supplemental Table 1. https://thejns.org/doi/suppl/10.3171/ 2019.1.SPINE181025.

\section{Correspondence}

Peter Vajkoczy: Charité-Universitätsmedizin Berlin, Germany. peter.vajkoczy@charite.de. 